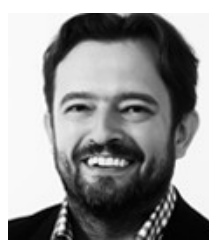

\section{Stephan M. Klinger}

Betriebswirt, Philosoph, Jurist und Familienvater; Lehrt an Porsche FernFH, TU Wien, WU Wien und JKU Linz

stephan.klinger@wu.ac.at

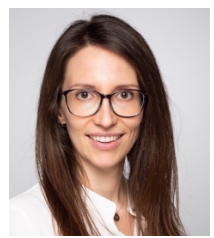

\section{Barbara Dimmel}

Bachelor in Betriebswirtschaft \& Wirtschaftspsychologie, selbstständig im Marketing-Bereich

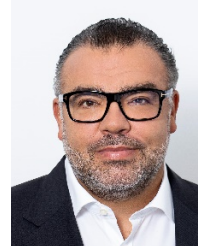

Dominik Damm

Partner bei Deloitte

Verantwortet den Bereich der

Bankenberatung

ddamm@deloitte.at

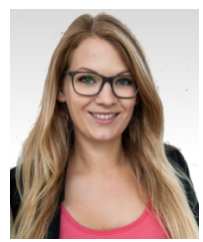

\section{Raphaela Franke}

Bachelor in Publizistikund Kommunikationswissenschaften, tätig in der Mediaplanung

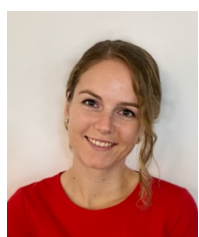

\section{Johanna Moser}

Bachelor in Marketing \& Sales, Trade Marketing Managerin

\title{
Junge Generationen denken Beratung neu
}

Studierende analysieren die Personalstrategien einer Unternehmensberatung auf Fit mit Bedürfnissen der Generationen $Y$ und $Z$

Der gegenwärtige Beitrag ${ }^{1}$ dokumentiert einen Praxis-Theorie-Transfer im Rahmen eines Lehrveranstaltungsprojekts an der Ferdinand Porsche FernFH. Das Ambidextrie-orientierte Beratungsunternehmen Deloitte lud Studierende ein, die eigene Personalstrategie auf den Fit mit den Bedürfnissen und Erwartungen der Generationen $Y$ (Geburtsjahre 1980-1995) und Z (Geburtsjahr nach 1995) auf Basis des gesamten Webauftritts zu analysieren und diesbezügliche Empfehlungen auszusprechen. In den Ergebnissen der studentischen Gruppen zeigen sich die Bedeutung von Werten wie Konsistenz, Transparenz, aktive und zielgruppengerechte Interaktion sowie die Erwartung an Offenheit und Ehrlichkeit abseits weichgezeichneter Semantik. 
„When you think back on your life; and you're 95, a hundred years old; And you look back over the course of your lifetime, you're not gonna think; I wish I owned a better phone, I wish I spent more time on the internet, I wish I spent more time at work or sleeping; It's not gonna be any of those kinds of things - It's gonna be I wish I spent more time with the people I love." Damit bringt der israelische Musiker Dennis Lloyd eine Sinnhaltung auf den Punkt: Workload mit Freuden und Freunden, Sinn- und Wachstumsorientiert sowie einem Schuss Leichtigkeit zu denken.

Eine solche Grundeinstellung wird gemeinhin den VertreterInnen der Generationen $Y$ und $Z$ zugeschrieben, zumal sie Leistungsbereitschaft, Entwicklungsbedürfnis und Sinnstiftung mitbringen und sich auch in Bewerbungsgesprächen nicht primär als aufopferungsbereite Humanressourcen präsentieren, sondern in Gehaltsverhandlungen ebenso selbstbewusst Fragen nach der Diversitätspolitik des Unternehmens oder nach Work-Life-Balance stellen. Das kann bei Unternehmen, welche eine gewachsene, auf Leistung, Karriere und monetäre Anreize ausgerichtete Personalstrategie (hohe Einsatzerwartungen verbunden mit einer „Up-or-Out Strategie“) verfolgen, für Irritationen sorgen. Der gegenständliche Artikel dokumentiert ein Projekt, in welchem berufstätige Studierende die Personalstrategie einer Unternehmensberatungsfirma analysieren und Handlungsempfehlungen für den Fit mit den Erwartungen der Generationen $Y$ und Z erarbeiten.

\section{Konzeptioneller Hintergrund}

Deloitte ist ein internationales Unternehmen und erbringt Dienstleistungen in den Bereichen Wirtschaftsprüfung, Steuerberatung, Financial Advisory, Risk Advisory und Consulting für Unternehmen aus allen Wirtschaftsbereichen. In seinem weltweiten Netzwerk von Mitgliedsgesellschaften in mehr als 150 Ländern sind über 300.000 MitarbeiterInnen tätig. Mit mehr als 1500 MitarbeiterInnen an 13 Standorten zählt Deloitte Österreich zu den führenden Anbietern von Professional Services. Dominik Damm ist Partner der Deloitte Financial Services Industry Advisory und begleitet mit seinem Team von über 30 ExpertInnen primär österreichische und international agierende Banken in wesentlichen regulatorischen, risikotechnischen, prozessualen und betriebswirtschaftlich strategischen Fragestellungen.

In der täglichen Arbeit als Unternehmensberaterln sind die Themen der „Exploration“ und „Exploitation“ kontextuell sehr stark verwoben. In diesem Spannungsfeld sucht Deloitte laufend motivierte Talente, die durch ihre akademische Top-Ausbildung naturgemäß die entsprechenden fachlichen Kompetenzen mitbringen und diese auch stetig weiterentwickeln, aber auch Motivation, Kreativität, Respekt und Enthusiasmus in den Arbeitsalltag einbringen. Unternehmensberatung findet nah am Kunden statt und ist in den wenigsten Fällen ein 9-5-Job. Ein hohes Commitment für den erfolgreichen und qualitativ hochwertigen Abschluss eines Projekts sowie die Einhaltung von oftmals sehr engen Deadlines auch unter Zeitdruck sind für diesen Job unabdingbar. 
Vor diesem Erwartungshintergrund arbeitet Deloitte kontinuierlich an seiner Personalstrategie und den entsprechenden Rahmenbedingungen, wie beispielsweise ein spannendes und abwechslungsreiches Arbeitsumfeld, moderne Büroräumlichkeiten in attraktiver Lage, stetige und auch internationale Aus- und Weiterbildungsmöglichkeiten sowie schnelle Lerneffekte „on the Job“, spannende Karrieremöglichkeiten, die sich auch finanziell niederschlagen, flexible Arbeitszeit und -platzmodelle und natürlich auch die Stabilität eines international agierenden Unternehmens uvm. Gegenseitige Wertschätzung und Respekt, Transparenz in wesentlichen Entscheidungswegen, Diversität und Gleichbehandlung sind zudem Werte, für die Deloitte im täglichen Arbeitsumfeld steht. Unternehmen wie auch Deloitte sind geprägt von loyalen und langfristig orientierten MitarbeiterInnen, um auch weiterhin erfolgreich im Spannungsfeld der Exploration und der Exploitation bestehen zu können.

Um die Außenwirkung dieser Rahmenbedingungen aus den Perspektiven der Generationen $\mathrm{Y}$ und $\mathrm{Z}$ (die sich in den Erwartungshaltungen von früheren Generationen doch deutlicher unterscheiden) einschätzen zu lassen und - im Sinne eines „reverse mentorings" - Gestaltungs- und Handlungsempfehlungen aus dieser Sicht zu bekommen, hat sich Deloitte für die Teilnahme an diesem Projekt entschieden.

Die konkreten Fragstellungen lauteten,

- wie eine adäquate und zielorientierte Ansprache von geeigneten KandidatInnen noch effizienter gelingen kann; und

- welche Werte der jungen Generationen zukünftig noch integrativer in Einklang mit den Anforderungen eines Ambidextrie-orientierten Beratungsunternehmens gebracht werden können, um den Karriereplan zielgruppenorientierter zu adaptieren.

Die Studierenden wurden eingeladen, die Personalstrategie von Deloitte auf Basis sämtlicher im virtuellen Raum verfügbarer Informationen zu analysieren, mit den Erwartungen und Werten der Generationen $Y$ und $Z$ zu vergleichen und entsprechende kreative Empfehlungen und Verbesserungsvorschläge zu erarbeiten und vorzustellen. Der Form und Struktur der Ergebnisse wurden keine weiteren Grenzen gesetzt, sondern völlig den Studierenden überlassen, da auch dies bereits - so zeigt es die Erfahrung - die erste kritische Reflexion der eigenen Überlegungen unter Beweis stellt.

Die Studierendengruppen hatten für die eigenständige Abwicklung des Projekts mehrere Wochen Zeit und präsentierten die Ergebnisse im virtuellen Raum auf einer digitalen Pinnwand (Padlet). Schon die Präsentationsformen zeigten eine erhebliche kreative Bandbreite und umfassten textliche Abhandlungen, Präsentationen, animierte Employee-Journeys oder aufgezeichnete Zoom-Konferenzen. Inhaltlich beeindruckend waren neben der Kreativität in der Ergebnisdarstellung auch die in einigen Gruppen durchgeführte Tiefen- und Hintergrundrecherchen des gesamten Social Media-Auftritts von Deloitte, deren Ergebnisse in Folge gekonnt zu Handlungsempfehlungen gebündelt 
wurden. Deloitte hat sich mit allen Ergebnissen beschäftigt und von den besten Gruppen jene Studentinnengruppe zur Mitwirkung an gegenständlichem Publikationsprojekt eingeladen und ersucht, ihre Vorgehensweise und Ergebnisse im Folgenden vorzustellen.

\section{Vorgehensweise und Ergebnisse der studentischen Arbeitsgruppe}

Von Beginn an war klar, dieses Projekt würde herausfordernd, jedoch durch seinen Praxisbezug und den direkten Kontakt mit einem international agierenden Unternehmen wie Deloitte auch sehr spannend werden. Die Aufgabenstellung war grundsätzlich offengehalten, aber dennoch ist einem bewusst, dass ein Unternehmen wie Deloitte klare Vorstellungen von den Ergebnissen hat. Unter dem Arbeitstitel „XYZ meets Deloitte" wurde das Projekt innerhalb des vierköpfigen Projektteams gestartet.

Die Arbeitswelt verändert sich und auch deren Akteure unterstehen einem stetigen Wandel, der sich besonders bei der Betrachtung unterschiedlicher Generationen zeigt. Die Karrieregedanken der jungen Generation sind anders als früher und somit andere als die der führenden PartnerInnen bei Deloitte. Diese Aspekte sind beispielhaft für die vorherrschenden Rahmenbedingungen bei der Durchführung des Projektes. Am Anfang stand die Analyse der Zielgruppen, um deren Ansprüche und Werte, aber vor allem auch deren Unterschiedlichkeit zu verstehen.

Momentan sind gleichzeitig vier Generationen am Arbeitsmarkt tätig, wodurch sich Erwartungshaltungen und Führungsansprüche ändern, zumal die unterschiedlichen Generationen verschiedene Ansprüche und Bedürfnisse haben. Besonders die Generationen $\mathrm{Y}$ und $\mathrm{Z}$ werden noch eine beträchtliche Zeit im Arbeitsleben stehen und waren die von Deloitte angeführten Fokusgruppen - daher wurden diese beiden genauer analysiert.

\section{Generationen $Y$ und $Z$}

Die Generation Y präsentiert die derzeit am besten erforschte Generation², zumal ihre Mitglieder in den frühen 1980ern bis zur Mitte der 1990er Jahre geboren wurden. Sie sind sehr gut ausgebildet und nicht selten werden durch Auslandsaufenthalte zusätzliche Erfahrungen gesammelt. Arbeit und Leben verbinden zu können und somit eine gute Work-Life-Balance zu schaffen, wird für sie immer wichtiger. Abwechslung und Selbstverwirklichung sind das A und O. Diese Kohorte sucht immer einen Sinn in dem was sie tut, was bedeutet, dass sie durchaus bereit ist, mehr zu leisten und zu lernen, wenn sie ein Projekt und/oder ihre Tätigkeit als sinnvoll empfindet. Auf der anderen Seite fragt sie sich, worin der Sinn einer Tätigkeit überhaupt besteht, was dieser Generation den Namen Generation „Why“ eingebracht hat. Sie arbeitet am liebsten im Team, bevorzugt flache Hierarchien, wünscht sich Anerkennung und Feedback für ihre Leistungen, strebt aber nicht unbedingt nach Führungspositionen ${ }^{3}$. 
Um all diese Bedürfnisse befriedigen zu können, sucht die Generation nach einem/einer ArbeitgeberIn mit guter Reputation und Entwicklungsmöglichkeiten. Da sie in einer Zeit der Veränderung aufgewachsen ist, Dinge sich schnell verändert haben und es wenige bis keine Sicherheiten gibt, hat sie gelernt das Leben „einfach“ zu genießen. Dadurch sind die Mitglieder dieser Generation aber auch sprunghafter als ihre Vorgängerlnnen und bereit, den Arbeitsplatz schneller zu wechseln, wenn etwas nicht zu ihrer

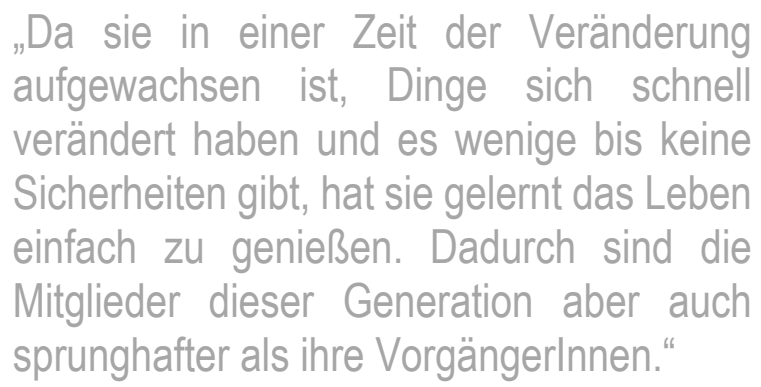

Zufriedenheit verläuft.

Außerdem wird viel Wert auf Diversität und Nachhaltigkeit gelegt. Als Digital Natives ist für sie mobiles Internet nicht mehr wegzudenken, durch Social Media ist diese Generation mit Menschen weltweit vernetzt ${ }^{4}$.

Mitglieder der Generation Z wurden ab Mitte der 1990er geboren und stehen somit schon ein paar Jahre im Arbeitsleben oder fassen gerade erst Fuß am Arbeitsmarkt. Sie sind ebenfalls sehr gut ausgebildet und Arbeit wird als Teil des Lebens gesehen. Sie streben nach Ansehen und guten Referenzen für den Lebenslauf. Work-Life-Balance ist ein absolutes Muss - so wie der Wunsch, Karriere auch in Teilzeit zu machen. Diese Kohorte möchte die hohen Erwartungen ihrer Eltern erfüllen und Erfolg, Karriere, Sicherheit und Stabilität werden wieder wichtiger. Auch diese Generation möchte unmittelbares Feedback für ihre Leistungen erhalten. Flexibles Arbeiten wird als Selbstverständlichkeit gesehen, was die moderne Technologie auch von nahezu überall ermöglicht. Die Generation folgt der Devise "Teamwork makes the dream work". Diversität und Gleichberechtigung nehmen einen wichtigen Platz ein und werden vom potenziellen Arbeitgeber erwartet, auch Nachhaltigkeit ist von sehr hoher Bedeutung. Diese Menschen sind „always on" - auf Social Media sind sie sehr gut vernetzt, auch Nachrichten werden hier konsumiert. Die Generation ist mit den neuesten Technologien aufgewachsen und erwartet das auch von ihrem Arbeitsplatz ${ }^{5}$.

\section{Die Welt von Deloitte}

Die auf diesen Ergebnissen fußende Analyse von Deloitte aus rein externer Sicht durchführen zu müssen, war in diesem Fall sogar ein Vorteil. Als externe Beobachterinnen hat die Gruppe die Perspektive eines/ $r$ möglichen externen KandidatIn eingenommen. Der Fokus wurde - den externen Möglichkeiten entsprechend - auf den Außenauftritt von Deloitte gelegt. In die Analyse miteinbezogen wurden: 
- die firmeneigene Website ${ }^{6}$

- mehrere Social Media Plattformen wie LinkedIn, Xing, Twitter, Instagram

- die Karriere-Plattformen Karriere.at, Kununu und Watchado

Die vorhandenen Inhalte auf diversen Plattformen wurden zuerst auf Vollständigkeit und Stringenz aus Sicht eines/r möglichen externen KandidatIn beurteilt. Anschließend konnte das sich ergebende Gesamtbild von Deloitte mit den Anforderungen der Generationen $Y$ und $Z$ abgeglichen werden, woraus sich abschließend konkrete Handlungsempfehlungen ableiten ließen.

Zusammengefasst liefert die durchgeführte Analyse die folgenden Ergebnisse:

Es wurde der Eindruck gewonnen, dass Deloitte durchaus ein sicherer Arbeitgeber zu sein scheint, auch bedingt durch das traditionell-konservative Auftreten. Deloitte präsentiert sich überdies als etabliertes, internationales Unternehmen, das über eine moderne Infrastruktur verfügt. Der Vorteil eines sehr zentral gelegenen Büros sowie unterschiedliche Weiterbildungsmöglichkeiten werden hervorgehoben. Diese Weiterbildung - das „am Puls der Zeit bleiben“ - wird von den MitarbeiterInnen aber auch verlangt - wie die Generationsanalyse gezeigt hat. Neben attraktiver Weiterbildung wird auch eine Vielzahl an Fringe Benefits geboten, welche die KandidatInnen überzeugen sollen.

Es scheint jedoch auch so, dass Arbeiten bei Deloitte kein „Zuckerschlecken“ wäre: In diversen Videos und Statements auf den einbezogenen Plattformen und auch in der anfänglichen Videobotschaft wurden immer wieder Überstunden und „Peak Zeiten“ erwähnt. Die entstehende Mehrleistung soll durch spannende Aufgabengebiete und mittels Zusammenarbeit mit interessanten Kundlnnen aufgewogen werden. Diese interessanten Aufgabengebiete bilden die Grundlage der sinnstiftenden Arbeit und der Möglichkeit des Aufbaus eines großen Netzwerks.

Der Außenauftritt erweckt zudem den Eindruck, dass Deloitte von hierarchischen Strukturen geprägt ist, die aussichtsreiche Karrieremöglichkeiten bieten, für deren Erreichen aber tatsächlich Einiges geleistet werden muss. Einige Berichte sprechen im Zusammenhang mit großer Leistung, folglich auch von entsprechendem Verzicht auf anderen Ebenen.

Nach der detaillierten Analyse blieben schließlich vier aus Sicht der Generationen $Y$ und Z offene Fragen zurück:

1. Wie schafft es Deloitte in den oft erwähnten „Peak Zeiten“ trotzdem eine gewisse Flexibilität und Work-Life-Balance zu bieten?

2. Gelingt mit den aktuell eingesetzten Medien und dem gewählten Content eine zielgruppengerechte konsistente Ansprache?

3. Wie wird die Selbstverwirklichung der jungen Talente gefördert? 
4. Wie groß werden die (doch) sehr wichtigen Themen Nachhaltigkeit und Diversität geschrieben?

Die dabei erarbeiteten Ergebnisse und Handlungsempfehlungen werden nun im folgenden Abschnitt dargelegt, um aufzuzeigen, wie man eine Brücke schaffen und so die neuen Werte der jungen Generationen mit den Anforderungen des Ambidextrieorientierten Unternehmensberatungs-Unternehmens Deloitte in Einklang bringen könnte.

\section{Ergebnisse und Empfehlungen}

Um die Ergebnisse und Empfehlungen darzulegen, wird der Fokus auf vier, für die Personalstrategie eines Unternehmens essenzielle, Aspekte gelegt: Den Auftritt von Deloitte nach außen und damit einhergehend die Ansprache von potenziellen neuen MitarbeiterInnen sowie die Themen Gestaltung des Karriereplans und MitarbeiterInnen-Bindung. Hierfür wird zum einen auf die Ergebnisse der Recherche zurückgegriffen und zum anderen wurden persönliche Erfahrungen aus dem Arbeitsalltag und daraus resultierende Ideen eingebracht.

\section{Auftritt und Ansprache}

Die Findings und daraus abgeleitete Empfehlungen hinsichtlich des Außenauftritts von Deloitte sowie der zielgruppengerechten Ansprache werden der Übersichtlichkeit halber in WAS (Content), WIE (Ansprache) und WO (welche Kanäle) aufgegliedert.

Beim Außenauftritt ist es entscheidend, dass potenzielle Mitarbeiterlnnen ein Bild von der zukünftigen Arbeitsumgebung bekommen können. Daher wurde der Content, den diverse Plattformen über Deloitte bieten, dahingehend genauer unter die Lupe genommen. Da allerdings (vermutlich auch aufgrund Vertraulichkeit) lediglich spärliche Informationen über den Arbeitsalltag selbst ausfindig "Was nicht 9-5 ist, wird auch niemals 9-5 werden - und das ist auch absolut in Ordnung. Seien Sie ehrlich und zeigen Sie transparent, was Deloitte zu bieten hat." hierauf gleich die erste Empfehlung. Um gezielt auf die Ansprüche der Generationen $Y$ und $Z$ einzugehen, wird vorgeschlagen, kurze Unternehmensvideos zu kreieren, in welchen ein Blick ins Office, also hinter die Kulissen, vermittelt wird. Dabei gilt es außerdem den Fokus auf die Teamarbeit sowie das Arbeiten auf Augenhöhe zu rücken, da es sich hierbei um zwei Aspekte handelt, die den jungen Generationen durchaus wichtig sind.

Auch das Thema Flexibilität wird von den Generationen $Y$ und Z großgeschrieben - wenn nicht sogar verlangt. Da dieser Begriff zugegebenermaßen sehr weit ausgelegt werden kann, gilt es hier klar zu kommunizieren, was unter Flexibilität konkret verstanden wird (wie etwa das Arbeiten von überall, Homeoffice-Möglichkeiten oder flexible 
Arbeitszeiten und eine gute Work Life Balance). Darüber hinaus sollten vor allem auch die Werte der Generationen Y und Z, wie Diversität, Nachhaltigkeit und soziale Themen, wie auch der Deloitte Future Fund, noch intensiver kommuniziert werden.

Bei der Kommunikation, für die Deloitte unterschiedliche Kanäle nutzt, ist es von Bedeutung, auch kanalübergreifend konsistent und einheitlich zu bleiben (z.B. kein Gemisch aus Sie/Du-Ansprache). In Hinblick auf die zielgruppengerechte Ansprache der Generationen YZ wird empfohlen, hierbei das DU zu wählen. Auch in Bezug auf Diversität ist darauf zu achten, dass diese nicht nur hie und da zu finden ist, sondern einheitlich kommuniziert und offen dargelegt wird. Nur so kann ein roter Faden durchgezogen und ein einheitliches Bild generiert werden.

Um potenzielle ArbeitnehmerInnen anzuziehen, muss Deloitte auf sich aufmerksam machen sowie ein positives Bild in den Köpfen erzeugen. Da heißt es, neben dem Image eines etablierten traditionellen Unternehmens auch Modernität aufzuzeigen. Um das zu erreichen, wäre es notwendig, etwa hinsichtlich der Ansprache Maßnahmen zu setzen: Zielgruppengerechtes Texten, DU-Ansprache sowie ein professioneller und moderner Inhalt, also ansprechende Videos und Bilder. Der Fokus soll auf dem Menschen liegen, Stichwort „new ways of working“ und auch die interaktive Kommunikation sollte im Vordergrund stehen.

Die richtigen Kanäle hingegen nutzt Deloitte bereits. Denn es ist auf allen gängigen Kanälen, die die Generationen $Y$ und $Z$ verwenden, vertreten. Jetzt muss der Fokus darauf liegen, den Content auf den entsprechenden Kanal anzupassen, zielgruppengerecht zu kommunizieren und konsistent zu bleiben.

Durch die eben dargestellten Maßnahmen werden geeignete KandidatInnen der Generation YZ zielgerichteter angesprochen. Wenn diese dann im Unternehmen arbeiten, geht es gleich weiter mit dem nächsten wichtigen Aspekt - der Mitarbeiterlnnenbindung. Denn das kommunizierte Ziel vieler Unternehmen ist es ja, gute MitarbeiterInnen möglichst lange zu halten.

\section{Karriereplan und MitarbeiterInnen-Bindung}

Transparenz ist ein Buzzword, das in der heutigen Zeit nicht mehr wegzudenken ist. Nichts Geringeres erwarten sich die Generationen $Y$ und Z von einem Karriereplan. Für Unternehmen bedeutet dies, keine Zweifel darüber zu lassen, was von MitarbeiterInnen erfüllt werden muss, um die nächste Stufe der Karriereleiter zu erklimmen.

Neben dem Wissenstransfer im Arbeitsalltag, also der Weiterbildung „on the Job“, ist es von großer Bedeutung Mitgliedern der Generationen $Y$ und Z die Möglichkeit zu geben, sich persönlich weiterzuentwickeln ${ }^{7}$. Insbesondere, weil dies dazu beiträgt, dass MitarbeiterInnen Innovationsprozesse im Unternehmen anstoßen oder aktiv Ideen einbringen. Auf einer möglichst individuellen Basis sollte auch dies in den Karriereplan mit einbezogen werden. 
Im Kontext der verschiedenen Generationen, die bei Deloitte beschäftigt sind, ist es wichtig, ein Umfeld zu schaffen, in dem die Menschen nicht nur miteinander arbeiten, sondern auch voneinander lernen können. Die entsprechende Interaktion könnte in der Form eines Mentoring-Programms gefördert werden. Dabei sollte der Wissenstransfer aber nicht, wie üblich, ausschließlich von langjährigen MitarbeiterInnen hin zu Jüngeren stattfinden, sondern ebenso in die entgegengesetzte Richtung, etwa in Form eines "Reverse Mentorings". Von dem unvoreingenommenen Blick neuer, junger Mitarbeiterlnnen auf das „Business as usual“ bis hin zu deren Know-how in Sachen Neue Technologien können auch arrivierte BeraterInnen profitieren.

Abschließend ist Sinnstiftung ein bedeutender Aspekt für die Generationen Y und Z. Bei Deloitte kann dieser in der spannenden und abwechslungsreichen Projektarbeit für namhafte Unternehmen mit sehr diversen Anforderungen liegen. Um diesen Vorteil auszubauen, könnte man MitarbeiterInnen von Zeit zu Zeit einladen, sich aktiv für solche Projekte zu melden, die den individuellen Interessen und Weiterbildungsplänen entsprechen.

\section{Fortentwicklungen seitens Deloitte}

Folgende Entwicklungen, für welche die Lösungsvorschläge der Studierenden eine Bestätigung der laufenden und bestehenden Initiativen darstellt, können seitens Deloitte bereits geteilt werden:

- Neben den bereits vorhandenen Videos, in denen Deloitte MitarbeiterInnen zu ihrem Arbeitsalltag und ihrer Erfahrung bei Deloitte interviewt werden, legt Deloitte auf Instagram nun beispielsweise besonders Wert auf die Umsetzung von Videos, die Interessierten einen Einblick „hinter die Kulissen“ ermöglichen. Auch Teamvorstellungen für die unterschiedlichen Bereiche sind in Planung und man darf gespannt sein.

- Die Themen Diversität, Nachhaltigkeit und soziales Engagement werden künftig einen noch intensiveren Schwerpunkt in der internen wie externen Kommunikation von Deloitte haben, da sie tatsächlich im täglichen Arbeitsalltag von ausgesprochen hoher Bedeutung für Deloitte sind. Mit der länderübergreifenden Kampagne „All-IN for Inclusion“ haben im Herbst letzten Jahres die ersten Postings aufgezeigt, welchen Stellenwert Diversität und Inklusion bei unseren KollegInnen haben und aus welchen Erfahrungen sie schöpfen können.

- „Interaktion“ wurde als weiterer Denkanstoß genannt. Hier kann das Unternehmen ebenso bereits weitere Schritte kommunizieren - so wird eine zusätzliche Unterstützung zur Entwicklung der Mitarbeiterinnen und Mitarbeiter durch "Career Coaching" - Coaching durch Peers - geschaffen und für Führungskräfte testet das Unternehmen derzeit ein Führungskräfte- 
Feedbacktool mit begleitender Reflektion. Ebenso bieten immer mehr Abteilungen von Deloitte bereits ein aktives und tourliches Mentoring im Sinne eines Buddy-Konzeptes an, das sich bewusst von der fachlichen Zuständigkeit in der täglichen Arbeit abgrenzt und eine auch „emotionale Teameinbettung" sicherstellt.

- Trotz der - auf den ersten Blick und karrieretechnisch erforderlichen Hierarchiestruktur bei Deloitte - gestaltet sich die täglich Zusammenarbeit in den jeweiligen Teams ausgesprochen partnerschaftlich und respektvoll. Die Kommunikation erfolgt - unabhängig von den jeweiligen Berufslevels - stets auf Augenhöhe und bedingt ein gegenseitiges Zuhören. Gerade im Beratungsgeschäft von Deloitte ist zudem das „Du“ mittlerweile Usus und wird auch in den Inseraten immer häufiger gezielt eingesetzt.

\section{Zusammenfassendes Projektfazit}

Als wesentliche generalisierbare Lessons' learnt dieser intergenerationalen Betrachtung von Unternehmensberatung können festgehalten werden:

1. Schaffen Sie Konsistenz. Sowohl in der Ansprache junger Talente als auch im Umgang mit Mitarbeiterlnnen.

2. Sorgen Sie für Transparenz auf allen Ebenen, insbesondere in den Bereichen Karriereplanung und Kommunikation.

3. Legen Sie großen Wert auf Interaktion - innerhalb des Unternehmens generationsübergreifend und in der Ansprache potenzieller MitarbeiterInnen über alle Kanäle hinweg.

4. Betonen Sie Teamstrukturen, die sich zu gesellschaftlichen wie auch ethischen Werte bekennen und diese auch in der Zusammenarbeit leben.

5. Und vor allem: Seien Sie offen und ehrlich, wenn es um KandidatInnenansprache geht. Wenn etwas nicht 9-5 ist, wird es durch bloße Kommunikation auch niemals 9-5 werden und das ist absolut in Ordnung. Stellen Sie andere, existieren Vorteile in den Vordergrund und zeigen Sie durch ehrliche Kommunikation, dass sich Fleiß und intensiver Arbeitseinsatz lohnen.

Methodisch wurde das Projekt seitens der Studierenden als großartige Möglichkeit gesehen an einem realen Praxisfall bei einem renommierten Beratungsunternehmen mitzuwirken. Das Wissen, dass das erarbeitete Ergebnis tatsächlich gehört wird und einen Input zu kommenden Entwicklungen liefern kann, stellte eine zusätzliche Motivation dar. Die Erwartungs- und Einstellungsprofile der VertreterInnen der Generationen $Y$ und $Z$ berücksichtigend, könnten solche Kollaborationsmodelle auf Augenhöhe einen wesentlichen Stellhebel für das Involvement dieser Generationen darstellen.

Die Vertreter des Projektpartners Deloitte haben im Rückblick der Analysetiefe, der Kreativität der Ergebnisse sowie dem hohen Zeitaufwand, der offenkundig in die 
Ergebniserarbeitung und in den inhaltlichen und graphischen Feinschliff investiert wurden, nachhaltigen Respekt gezollt. Die meisten Gruppen haben ein Involvement in diesem Projekt gezeigt, welches weit über die Notenbedeutung dieses Projekts in der Lehrveranstaltung hinausgeht.

Es bestätigt sich, dass durch die offene Aufgabenstellung und dementsprechend unterschiedlichen Herangehensweisen der Gruppen bei der Analyse und Lösungsdarstellung ein bunter Strauß an wertvollen Erkenntnissen entstanden ist. Von der Bearbeitung einer Aufgabestellung wie der „Deloitte Challenge“ lässt sich wohl ableiten, dass die „freiheitsliebenden" Generationen $Y$ und Z mit wenigen, prägnant formulierten Angaben und viel Freiraum für die Lösungsentwicklung offensichtlich bessere Ergebnisse zu liefern vermögen als mit zahlreichen einschränkenden, wenngleich detaillierten Vorgaben.

\section{Conclusio}

Die Austrian Academy of Management \& Leadership verfolgt das Ziel eines befruchtenden und gleichberechtigten Austausches zwischen den Sphären Praxis und Theorie. Der vorgestellte Beitrag stellt ein Beispiel für die daraus erwachsenden Möglichkeiten dar: eine Themenstellung aus der unternehmerischen Praxis (Wie wird meine Personalstrategie von meinen zukünftigen Zielgruppen wahrgenommen?) wird von theoretisch-fundierten Studierenden aufgegriffen, die gleichzeitig Vertreterinnen dieser Zielgruppe sind (also auch darin praktische Vorerfahrung mitbringen) und in einem möglichst ergebnisoffenen Setting bearbeitet.

Was diese interessante Erarbeitung von Lösungsvorschlägen aller studentischen Gruppen im Projekt auch gezeigt hat, ist das latente Bedürfnis junger Generationen voneinander lernen zu können - fern von dominanten Hierarchiemustern drängt die Sehnsucht durch gemeinsame Interaktion an Unternehmenswerten zu arbeiten und persönliche Wertvorstellungen zu tradieren. Mentorenprogramme sehen nicht zwangsweise den erfahrenen Berater als Mentor vor - er kann auch als Mentee von einer jungen Beraterin in die digitalen Welten eingeführt werden. Gerade Social Media-Auftritte eines Unternehmens vermitteln, wie von den Kolleginnen sehr gut beschrieben, implizite Bilder, Werthaltungen und Einstellungen der existierenden Unternehmenskultur, die geeignete KandidatInnen anspricht und attrahiert - oder auch nicht. Unternehmen, die sich auf eine solche Perspektiventradierung von Sinngebung einlassen, können damit - quasi nebenbei - organisationale Change Prozesse einleiten.

Positiv betrachtet könnten die Sichtweisen und Wünsche der kommenden Generationen $Y, Z$ (und welche Buchstaben danach auch immer kommen werden), auch die Reduktion des eigenen Lebens auf Arbeit und Konsum als wesentliche Determinanten menschlicher Tätigkeit wieder erweitern. Die deutsch-amerikanische Philosophin Hannah Arendt hat in den 1950er Jahren die „Human Condition“ " 8 in folgende menschliche 
Grundtätigkeiten gegliedert: Arbeit, Herstellen und Handeln. Während „Arbeit“, dem biologischen Prozess des Körpers nachgebildet, an der bloßen Lebensnotwendigkeit orientiert ist, entspringt der menschliche Drang des „Herstellens“ dem Wissen um die eigene Vergänglichkeit und mündet darin Dinge herzustellen, die das eigene Leben überdauern (Sinngebung). Das „Handeln“ spielt sich zwischen den Individuen ab und verbindet diese Menschen. „I wish I spent more time with the people I love."

\section{Literatur}

1 Das Autorlnnenteam bedankt sich bei 2 ReviewerInnen für anregende und konstruktive Hinweise, sowie bei Katharina Wolf MA und Sabine Klinger BA MA für ihre Unterstützungen bei der Erstellung dieses Beitrags.

2 Siehe etwa Lutze, M.; Schaller, P.; Wüthrich, H. (2019). New Work - Zurück in die Zukunft der Motivation. ZfO, 6, 88. Jg., 356-362 bzw Grimm, A. (2016). Mitarbeiterbindung in der Generation Y - Empirische Studie zu Faktoren, die das Commitment junger Mitarbeiter fördern. ZfO, 1, 85. Jg., 45-50

3 Reisinger, S.; Lettner, N.; Friedl, A. (2019). Erfolg braucht Menschen - Wettbewerbsvorteile durch eine konsequente Mitarbeiterorientierung. ZfO, 5, 88.Jg., 322-328

4 Kolland, F. ; Wanka, A.; Bischof, C. \& Psihoda, S. (2015). Von den Baby Boomern zur Generation Y. Tipps für erfolgreiches Generationenmanagement. Online: https://www.wko.at/site/Charta-der-Vielfalt/Service/publikationen/Broschuere_BabyBoomer_PRINT_22-10-15.pdf [Abruf am 11.04.2021]

5 Mangelsdorf, M. (2015). Von Babyboomer bis Generation Z. Der richtige Umgang mit unterschiedlichen Generationen im Unternehmen. Gabal, Offenbach

6 https://www2.deloitte.com/at/de.html

7 Konlechner, S. \& Güttel, W. (2009). Kontinuierlicher Wandel mit Ambidexterity. ZfO, 1, 78. Jg., 45-53

8 Arendt, H. (1972): Vita activa oder „Vom tätigen Leben“; Piper, München

\section{Information zu den Autorlnnen}

MMMag. Dr. Stephan M. Klinger unterrichtet an der JKU Linz, der TU und WU Wien, der Ferdinand Porsche FernFH sowie an der FH des BFI Wien Themen wie Ethik, Verhandlungsmanagement, HRM und Veränderungsmanagement sowie Managing Generations and Technological Change.

MMag. Dominik Damm ist Partner der Deloitte Financial Services Industry Advisory und begleitet mit seinem Team von über 30 ExpertInnen primär österreichische und internationalagierende Banken in wesentlichen regulatorischen, risikotechnischen, prozessualen und betriebswirtschaftlich strategischen Fragestellungen.

Barbara Dimmel, BA absolvierte den Bachelorstudiengang Betriebswirtschaft \& Wirtschaftspsychologiean der Ferdinand Porsche FernFH und ist zurzeit Studierende des Masterstudiengangs Betriebswirtschaft \& Wirtschaftspsychologie an der Ferdinand Porsche FernFH.

Raphaela Franke, BA hat den Bakkalaureatsstudiengang Publizistik- und Kommunikationswissenschaften auf der Universität Wien abgeschlossen und studiert derzeit 
Stephan M. Klinger et al.

berufsbegleitend im Masterstudiengang Betriebswirtschaft und Wirtschaftspsychologie mit Schwerpunkt Marketing Management an der Ferdinand Porsche FernFH.

Nicole Hogn, BA hat den Bachelorstudiengang Unternehmensführung - Entrepreneurship an der FHWien der WKW abgeschlossen und ist zurzeit Studierende des Masterstudiengangs Betriebswirtschaft \& Wirtschaftspsychologie an der Ferdinand Porsche FernFH.

Johanna Moser, BA absolvierte den Bachelorstudiengang Marketing \& Sales an der FH Wien der WKW und studiert derzeit berufsbegleitend Betriebswirtschaft \& Wirtschaftspsychologie mit Schwerpunkt Marketing Management an der Ferdinand Porsche FernFH. 